\title{
¿POR QUÉ NO NOS GUSTAN ALGUNOS COMPAÑEROS? RAZONES DE LOS ESCOLARES DE PRIMERO Y SEGUNDO CURSO DE EDUCACIÓN PRIMARIA
}

\author{
Francisco J. García Bacete \\ Universitat Jaume I (Castellón). Grupo GREI \\ fgarcia@uji.es \\ Gonzalo Musitu Ochoa \\ Universidad Pablo Olavide (Sevilla). Grupo Lisis \\ Anna I. LLopis Llueca \\ Profesora de Educación Secundaria Jubilada. \\ Ex directora del Instituto IES Orriols (Valencia)
}

Fecha de Recepción: 26 Junio 2019

Fecha de Admisión: 25 Septiembre 2019

\section{RESUMEN}

El objetivo de este artículo es estudiar por qué a los niños/as de 6-7 años no les gustan otros compañeros/as de su misma aula. El trabajo profundiza en el fundamento y la definición de cada categoría de motivos de desagrado propuesta por García Bacete, Marande, Carrero y Musitu (2017), y en el significado de motivos específicos, lo que permite ser más precisos en la identificación de similitudes y diferencias en las comparaciones constantes motivo-motivo, motivo-categoría y categoría-categoría. Esto ha permitido construir un mapeo completo de los motivos y elaborar una guía que orienta de forma integrada la comprensión y la clasificación de los motivos en ocho categorías: conductas agresivas, conductas de dominancia, conductas sociales y escolares problemáticas, molestias al bienestar, identidad personal, identidad social, falta de intercambios y no sabe/no contesta. $Y$ aunque han emergido algunos cambios, el resultado refuerza la taxonomía original. De cada categoría se ofrece su definición, fundamentos y ejemplos representativos. Se concluye que los juicios de desagrado son diversos, suelen ser encubiertos, tienen base grupal y revelan fallos en la inclusión. Finalmente se ofrecen pautas para la prevención y la intervención en las aulas.

Palabras claves: razones de desagrado; teoría fundamentada; coste de conducta; identidad; indiferencia; educación primaria.

\section{ABSTRACT}

Why do we dislike some classmates? Reasons of first and second graders elementary school. The aim of this work is to study why children aged 6-7 do not like other classmates. The article goes in depth in the rationale and definition of each category of reasons for disliking proposed by García Bacete, Marande, Carrero and Musitu (2017), and in the meaning of specific reasons, so 


\section{¿POR QUÉ NO NOS GUSTAN ALGUNOS COMPAÑEROS? RAZONES DE LOS ESCOLARES DE PRIMERO Y SEGUNDO CURSO DE EDUCACIÓN PRIMARIA}

that we can identify more accurately similarities and differences in the constant comparisons reason-reason, reason-category and category-category. This has led to the construction of a complete mapping of the reasons and a guide to facilitate an integrated way of understanding and classifying the reasons in eight categories: aggressive behaviors, dominance behaviors, problematic social and school behaviors, inconvenience to well-being, personal identity, social identity, lack of exchanges and "do not know / no answer". Although some changes did emerge, the outcome reinforces the original taxonomy. A definition for each category, as well as theoretical basis and representative examples, are provided. The conclusion is that the motives for disliking are diverse and usually not put on display, that they have a group basis and reveal failures in inclusion. Finally, guidelines for prevention and intervention in classrooms are offered.

Keywords: reasons for disliking; grounded theory; cost of behavior; identity; indifference; elementary education.

\section{INTRODUCCIÓN}

Las relaciones entre iguales adquieren cada vez mayor importancia a lo largo de la educación primaria. Como señalan Bukowski, Motzoi y Meyer (2009), casi todos los niños dicen tener amigos y asocian esta relación con experiencias agradables. Casas (2010) encontró que estar con los amigos es la actividad favorita de los escolares españoles entre 7 y 16 años. Pero en las relaciones interpersonales a cualquier edad no solo intervienen los agrados y aceptaciones sino también los desagrados y los rechazos, y como se ha señalado reiteradamente (p.e., Bukowski, Sippola, Hoza, \& Newcomb, 2000) ambas dimensiones son funcionalmente diferentes, y ser objeto de desagrados no coincide con tener poca o baja aceptación.

Según Vallés y Vallés (2000) el desagrado es un sentimiento 0 actitud de disgusto 0 aversión hacia una persona, situación u objeto. La expresión "no me gusta", "dislike" en inglés, sintetiza de forma clara lo que significa el rechazo o desagrado. Así como la atracción sugiere la intención de acercarse, el disgusto y la aversión conlleva la predisposición a interrumpir el contacto 0 alejarse de alguien 0 de algo (Redorta, Obiols, y Bisquerra, 2006). Además, como señalan diversos autores el rechazo interpersonal es una experiencia adversa, común e inevitable (Leary, 2001).

La propia naturaleza del rechazo y las características de la vida social dan apoyo a la inevitabilidad del rechazo. Como emoción y/o actitud negativa sirve para hacer frente de forma inmediata a acontecimientos valorados como una amenaza, pérdida, frustración, bloqueo, dificultad,... Comparativamente con las emociones positivas son más intensas y duraderas y afectan más (Bisquerra, 2015). En las relaciones sociales necesariamente hay que hacer elecciones porque aceptar a todos requeriría de mucho tiempo y energía (Leary, 2001), de modo que a quienes no entran en nuestro nicho relacional se les ignora, se les evita o se les rechaza. Sin embargo, las personas durante las interacciones sociales en muchas ocasiones no comunican sus opiniones 0 emociones negativas hacia sus interlocutores, en un intento para autoprotegerse de posibles reacciones negativas o para evitar la pérdida de privilegios (Bellmore \& Cillessen, 2003), lo que dificulta la posibilidad de revertir la situación.

Pero, además, las simpatías y antipatías, amistades y enemistades suelen surgir en comunidades y grupos más grandes y estables, como es el caso de las aulas en los colegios. Ser alumno de una determinada aula y tener los mismos compañeros es una situación que se va a extender durante la mayor parte del periodo escolar para la mayoría de escolares, lo que supone una ecología social muy exigente. La escuela no debe evitar los desagrados, que como hemos visto son naturales y funcionales, ni decir a los niños y niñas qué compañeros han de ser sus amigos, pero la escuela es un contexto especial, comprometido con la inclusión, en donde caben las diferencias, por lo que hacer 
visibles los desagrados, que todos y todas puedan expresarlos con naturalidad, estar atenta a lo que sucede en las interacciones interpersonales y en los grupos, y sobretodo promover el máximo de oportunidades para relacionarse y aprovechar la funcionalidad de los desagrados son obligaciones de la escuela. Para ayudar a este fin nos hemos propuesto conocer mejor la naturaleza de los desagrados. Para ello revisaremos y describiremos extensamente cada una de las razones de desagrado identificadas por García Bacete, Marande, Carrero y Musitu (2017) y haremos propuestas para trabajar en las escuelas las emociones y actitudes negativas. Antes, analizaremos las características del rechazo entre iguales, y cómo estas sugieren el método adecuado para estudiar las razones de rechazo.

\section{RECHAZO ENTRE IGUALES EN LA INFANCIA}

El intercambio de desagrados 0 afectos negativos entre compañeros es una experiencia adversa común desde el inicio de la escolaridad. El 95.5\% de niños y niñas de 6-7 años nominan negativamente a algún compañero cuando se les solicita que nombren los niños o niñas de su clase que no le gustan y el $85.5 \%$ de los niños y niñas reciben al menos una nominación negativa de otro compañero (García Bacete et al., 2017). Intuitivamente, por rechazo se entiende el desagrado que un niño 0 niña siente hacia otro compañero o compañera, o hacia algo que hace o que dice. Se habla de rechazo entre iguales cuando no es sólo un rechazador sino un número significativo de iguales del aula quienes sienten el desagrado hacia un niño o niña. Tradicionalmente la psicología del desarrollo preocupada por los importantes efectos negativos del rechazo se ha interesado más por los niños o niñas rechazados o no queridos que por los rechazadores, lo que ha enfatizado el foco en los correlatos del rechazo (Bierman, 2004). Bierman (2004) describió a los niños y niñas rechazados diciendo que pueden ser más discutidores, perturbadores y agresivos, socialmente más torpes e insensibles, menos hábiles para participar en juegos prosociales y / 0 tienen más interacciones negativas con los maestros que sus compañeros. Este énfasis en las conductas inadecuadas, deficientes o incompetentes de los rechazados apoya la idea de que los rechazados son responsables de esta situación (McDougall, Hymel, Vaillancourt, \& Mercer, 2001). Sin embargo, otros autores no han encontrado esta asociación. Por ejemplo, Chang (2004) encontró que algunos niños rechazados eran populares y miembros centrales de sus grupos. Este resultado y otros similares parecen indicar que el rechazo no es una característica del niño rechazado o de su comportamiento, sino que está en función de aquellos que le rechazan. Sin embargo, pocos estudios se han interesado por los rechazadores y cuando lo han hecho ha sido para describir las conductas explícitas de rechazo (Bierman, 2004), olvidando que antes es necesario haber realizado una evaluación o interpretación negativa de las conductas o personas a las que se rechaza.

McDougall et al. (2001, p. 214) se refirió al rechazo entre iguales como "el desagrado entre compañeros que puede estar o no acompañado por un grado variable de victimización, exclusión 0 aislamiento intencional de las actividades". Esta definición incluye las características esenciales del rechazo entre iguales.

En primer lugar se afirma que hablar de rechazo es sinónimo de hablar de desagrados. En sentido estricto, de muchos desagrados, de aquí nuestro interés en comprender los desagrados como elementos básicos sobre los que se construye el rechazo, y en particular, por las explicaciones que los niños y niñas dan de sus rechazos. Como hemos comentado los desagrados son actitudes o sentimientos negativos. Lo más característico de los sentimientos es su dimensión agrado-desagrado, según proporcione bienestar o malestar, y la esencia de las actitudes es la posición a favor 0 en contra (Redorta et al., 2006). Los sentimientos informan sobre cómo se siente una persona y de sus intenciones y las actitudes de cómo piensa, lo que pone en primer plano que el rechazo es una eva- 


\section{¿POR QUÉ NO NOS GUSTAN ALGUNOS COMPAÑEROS? RAZONES DE LOS ESCOLARES DE PRIMERO Y SEGUNDO CURSO DE EDUCACIÓN PRIMARIA}

luación o interpretación por parte de quienes rechazan. Leary (2001) afirmó que lo característico de todas las formas de rechazo interpersonal es que se trata de una evaluación relacional baja por la que una persona considera que la relación con otra persona no es valiosa, importante o próxima. En casos extremos considera que la relación no tiene ningún valor, incluso que es negativa y despreciable, y en casos más ligeros se considera que la relación es mínima y de esta forma decide invertir poco o nada en mantener la relación.

Segundo, al señalar que el rechazo no consiste necesariamente en la victimización, exclusión, o aislamiento del rechazado se desea diferenciar el rechazo de las conductas o expresiones de rechazo, e indirectamente afirmar que en muchas ocasiones el rechazo es encubierto, esto es, que no tiene visibilidad externa o que no se comunica. Leary (2005) afirmó que el rechazo se da en forma de evaluaciones negativas encubiertas más que en forma de conductas manifiestas dirigidas hacia un individuo. En efecto, la investigación señala que el $82 \%$ de los niños victimizados son rechazados, pero solo el $38 \%$ de los niños y niñas rechazados son victimizados (Schuster, 2001). Este carácter encubierto 0 de no visibilidad de los desagrados se confirma tanto desde la perspectiva de Ios rechazadores como desde la perspectiva de los rechazados. El $51 \%$ de los rechazadores de cuarto curso informan que los compañeros que no les gustan lo desconocen (Moliner, 2018). Afirmación que suelen complementar diciendo que ellos ni hacen ni dicen nada para que lo sepa, esto es, que lo ocultan. Desde el punto de vista del rechazado, García Bacete, Marande, Schneider y Cillessen (2019b) han mostrado que el 95\% de los niños y niñas de 6-7 años comenten algún tipo de error en la identificación de sus rechazadores y que el 50\% no acierta ninguno de sus posibles rechazadores.

Tercero, al hablar en términos de compañeros se indica que el rechazo se produce en el contexto de un grupo, entre iguales que se conocen, que comparten una historia, que generalmente continuará, como opuesto a niños desconocidos (Doosje, Spears, \& Ellemers, 2002). Cabe pensar, pues, que ciertas características de los grupos, de las aulas, puede influir en los miembros del grupo en lo que gusta y lo que no (Nesdale, 2011). Además, como para hablar de rechazo entre iguales se requiere que un número significativo de compañeros evalúen negativamente a otro compañero, como sugirieron Berger y Dijkstra (2013) se puede pensar que existe un cierto consenso o regularidades en los juicios de los rechazadores en un grupo. En esta dirección, Leary (2001) indicó que una mirada amplia permitía descubrir regularidades evidentes en los patrones de rechazo (p.e., no suelen gustar quienes no contribuyen a las metas comunes) 0 que ciertos grupos son potencialmente más rechazados que otros (p.e., los que tienen una apariencia diferente). Y como la vida escolar de los niños se caracteriza por estar permanentemente agrupados, apoya que varios autores afirmen que un modelo de rechazo entre iguales solo estará completo cuando se comprendan las influencias del contexto social (García Bacete et al., 2017; Mikami, Lerner, \& Lun, 2010) y que lo que parece ser un rechazo interpersonal podría estar reflejando en realidad un rechazo grupal (Killen, Mulvey, \& Hitti, 2013).

En conclusión, dado el carácter interpretativo y a menudo encubierto de los desagrados, y el que se produzca entre compañeros conocidos entremezclando lo interpersonal y lo grupal, el estudio de las razones requiere centrarse en quién rechaza y llevar a cabo el estudio en los entornos ecológicos donde tienen lugar las interacciones entre compañeros, como son las aulas de los colegios.

Los antecedentes empíricos más próximos son los trabajos de Smith (1950), Feinberg, Smith y Schmidt (1958), Hayes, Gershman y Halteman (1996), Monjas, Sureda y García Bacete (2008), Di Napoli (2017) y García Bacete et al. (2017). Estos estudios representan una importante contribución, ya que analizaron las razones en situaciones donde los niños rechazadores interactuaban a diario con los rechazados, que son muy diferentes de las situaciones hipotéticas con protagonistas anóni- 
mos sin antecedentes ni futuro que suelen ser habituales en la investigación (Doosje et al., 2002). Sin embargo, las razones del rechazo en estos estudios se presentan de manera descriptiva sin profundizar en las interconexiones entre categorías ni entre los motivos dentro de cada categoría, e interpretan las razones en gran medida en términos del comportamiento del niño rechazado sin tener en cuenta el contexto de grupo de rechazador y rechazado.

En el trabajo de García Bacete et al. (2017) se aplica la teoría fundamentada de modo más sistemático tratando de avanzar en una formulación teórica capaz de explicar los datos. Como resultado se elaboró una taxonomía de los motivos de desagrado. La taxonomía está formada por siete categorías sustantivas, tres supracategorías y tres categorías contextuales. Las categorías sustantivas se refieren al significado del motivo: Conductas agresivas, dominancia, conductas sociales y escolares problemáticas, molestias al bienestar, identidad personal, identidad social, y falta de contacto y/o familiaridad. Las supracategorías son variables latentes capaces de explicar las categorías sustantivas de modo integrado: las conductas desviadas de las normas interpersonales y grupales, las identidades personales y sociales y la indiferencia entre compañeros (ver Figura 1). Las categorías de contexto son transversales a las sustantivas y se refieren a las condiciones en las que se produce el rechazo, como son los participantes, el impacto y el papel de intérprete u observador que juega el rechazador.

Con el objetivo de describir del modo más exhaustivo y preciso cada categoría sustantiva, y señalar los cambios si los hubiera, este trabajo se instala en la fase teórica del método, y prosigue con la comparación constante indicador-indicador, indicador-categoría y categoría-categoría, usando los mismos indicadores que García Bacete et al. (2017), profundizando en las definiciones y fundamentos de las categorías y en el ajuste de los indicadores a dichas definiciones.

Figura 1. Razones de desagrado. Vías que conducen al rechazo (adaptado de García Bacete et al. 2017)

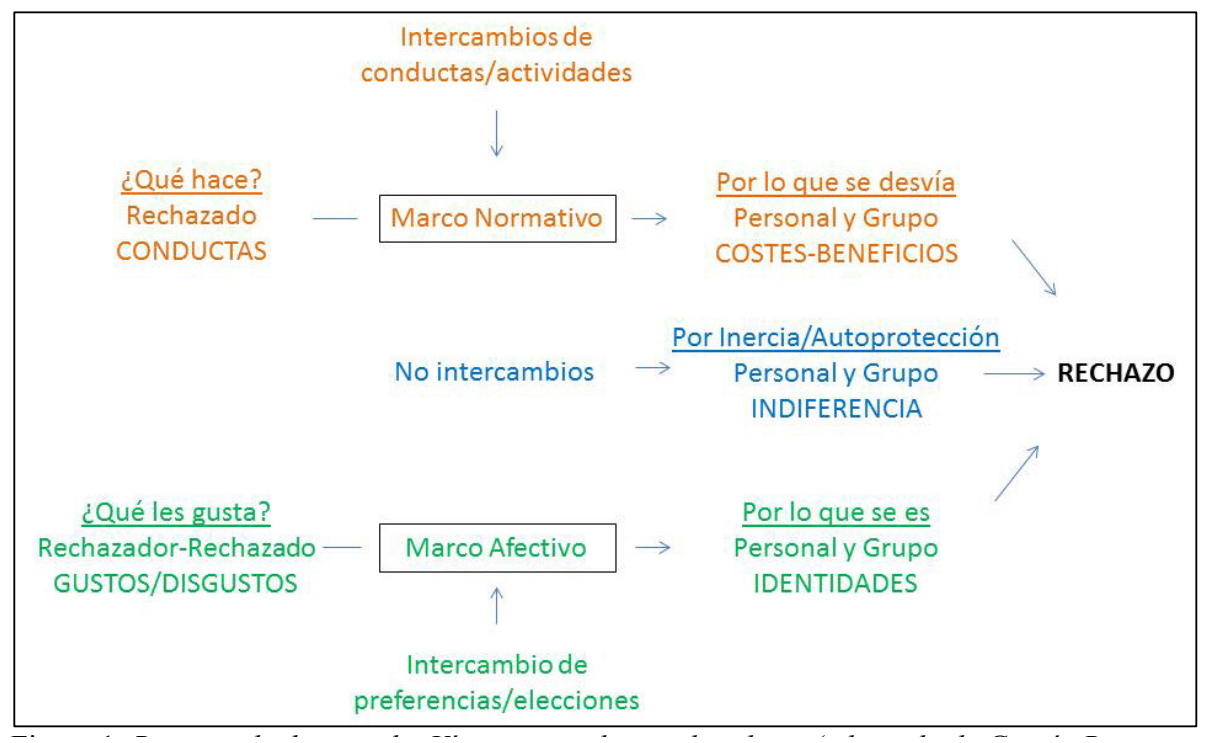




\section{¿POR QUÉ NO NOS GUSTAN ALGUNOS COMPAÑEROS? RAZONES DE LOS ESCOLARES DE PRIMERO Y SEGUNDO CURSO DE EDUCACIÓN PRIMARIA}

\section{METODOLOGÍA}

\section{Participantes}

Para realizar la investigación se llevó a cabo un muestreo incidental por accesibilidad que permitió seleccionar todas las aulas de primero y segundo de Educación Primaria (40 aulas) de 13 centros públicos de Educación Primaria en zonas urbanas de nivel socioeconómico medio, cercanas a las cuatro universidades participantes en el estudio. De los 939 sujetos iniciales, 86 alumnos no contestaron, 46 causaron baja en el colegio durante el estudio y 40 no emitieron nominaciones de rechazo. Los 853 informantes proporcionaron 2934 razones por las que no les gustaban algunos de sus compañeros. El $69 \%$ de los informantes es de primer curso y el $31 \%$ de segundo, el $84 \%$ tiene 6 años (Media $=6.76$ años, DT= 0.87), 50\% de cada sexo. La participación en el estudio fue voluntaria. Las familias dieron su consentimiento informado por escrito.

\section{Instrumentos}

La información recopilada se extrajo mediante entrevistas individuales semiestructuradas en las que los niños y niñas contestaban dos preguntas extraídas del cuestionario sociométrico elaborado por García Bacete y cols. (2017): 1) ¿De todas las niñas y niños de esta clase con qué niños o niñas no te gusta estar? y 2) ¿Por qué no te gusta "..."? (Nombre de cada uno de los niños-as nombrados en la pregunta 1). Se compiló una lista con las 3009 razones verbalizadas por los alumnos para explicar el rechazo hacia sus compañeros y se recurrió a la teoría fundamentada como estrategia de análisis (Carrero, Soriano, y Trinidad, 2012). Para evitar que la opinión o visión de determinados niños quede sobrerrepresentada, en los análisis cuantitativos se utilizó un máximo de 5 razones por informante. El total de motivos queda en 2678.

\section{Procedimiento}

Las consideraciones conceptuales sobre el rechazo entre iguales comentadas en la introducción conllevan importantes decisiones metodológicas para el estudio de los motivos de desagrado. La primera es que la principal fuente de información no son los rechazados, sino quienes rechazan. Solo preguntando a los rechazadores estaremos seguros de la existencia y del tipo de desagrado y de las razones que le llevan a no querer 0 a no gustarle un compañero. Cada persona es la más indicada para informar de sus sentimientos y pensamientos. La segunda decisión afecta al método de recogida de la información. Hemos preferido el método de preguntas abiertas y directas al rechazador porque son más significativas y permiten respuestas más espontáneas (Elig, \& Frize, 1979), que pedir a los niños que seleccionen o valoren motivos predeterminados en situaciones hipotéticas, y a veces extrañas. La tercera decisión consiste en realizar la investigación en contextos significativos generadores de la información, esto es, con iguales que comparten cotidianamente un grupo, un aula,..., recuperando así la propuesta original de Moreno (1934), "Ios participantes en un sociograma han de estar juntos en función de alguna norma, como por ejemplo un aula,... deben responder con un alto grado de espontaneidad,... preguntas referidas a fenómenos auténticos". En nuestro caso preguntamos a cada niño/a qué compañeros de su clase no le gustan y las razones concretas por las que no le gusta cada uno de los compañeros que nombra. La cuarta decisión se refiere a la estrategia de análisis de las respuestas. En este caso pensamos que se necesita un enfoque cualitativo para comprender las razones que hacen que los niños no les guste o rechacen a algunos compañeros. La metodología fundamentada desarrollada por Glaser y Strauss en 1967 ha sido nuestra elección porque ha demostrado ser capaz de generar categorías conceptuales básicas a partir de datos que explican los procesos que ocurren en situaciones sociales complejas (Carrero et al., 2012). Desde esta perspectiva, no es necesaria una taxonomía predeterminada de las posibles razo- 
nes ni un marco explicativo previo para interpretar las razones. En lugar de eso, la teoría fundamentada parte de los datos proporcionados por los participantes sociales y, al aplicar la comparación constante de los incidentes encontrados (las razones dadas por los rechazadores), hace emerger categorías sustantivas que se comparan con los nuevos incidentes. Estas comparaciones sucesivas continúan hasta lograr la saturación teórica de los datos proporcionados por los participantes. Este proceso produce una estructura subyacente que explica la variabilidad de las razones aportadas por los participantes. Para más información de la metodología y de las fases de categorización consultar Carrero et al. (2012) y García Bacete et al. (2017).

\section{RESULTADOS}

\section{Comparativa con la Taxonomía propuesta por García Bacete et al. (2017)}

La estructura de la taxonomía que se ha identificado sigue siendo la misma que elaboraron García Bacete et al. (2017), pero se han producido algunas modificaciones. A continuación comentamos brevemente las más significativas.

Entre supracategorías o vías explicativas del desagrado. 1. Coste de conducta vs. Identidad. Tomamos conciencia de que si las razones de la supracategoría coste de conducta se refieren a conductas desviadas del rechazador, lo específico de las razones de la supracategoría identidad es que son motivos centrados en las relaciones y exclusiones entre rechazador y rechazado. En esta segunda supracategoría prima el componente relacional (juego, amistad, conflicto, exclusión, reputación,...), frente al componente conductual de la primera. 2. Identidad vs. Indiferencia. En la supracategoría indiferencia se expresan hechos, se afirma la existencia de poco o ningún contacto ente los niños (No nos conocemos,..., no nos vemos... no vamos... no jugamos..., no somos amigos...), mientras que la supracategoría identidad se expresan relaciones que se mantienen o en la voluntad de no tenerlas o de excluirse. 3. Categoría No sabe-no contesta. Las respuestas "no sabeno contesta" dejan de ser vistas como datos perdidos y se decide que se integren como una nueva categoría en la supracategoría indiferencia, porque la ausencia de motivo que expresan estas respuestas comparte significado con los motivos de la categoría falta de contactos y/o familiaridad, esto es, el desconocimiento, el desinterés, la inercia, la sinrazón.

Entre categorías. Emergen algunas propiedades que ayudan a diferenciar entre categorías, y permiten una clasificación más precisa de los motivos. Algunos ejemplos: 4. Conductas sociales y escolares problemáticas vs. Molestias al bienestar. A diferencia de la categoría conductas problemáticas, en la categoría molestias el énfasis no se pone tanto en qué hace el rechazado, lo que a veces ni se nombra, si no en el efecto que provoca la conducta del rechazado en el rechazador. 5. Conductas sociales y escolares problemáticas vs. Conductas agresivas. Se decide que para que una conducta se clasifique como agresión no sólo es necesaria la intención de hacer daño, sino que el daño se cause a personas. Por ejemplo, "le dice cosas feas a mi amiga" se clasifica en agresión verbaly "se porta mal conmigo" en intimidación. Sin embargo los motivos "dice cosas feas" o "se porta mal" se clasifican en la categoría conductas problemáticas.

Entre indicadores. Algunos motivos concretos cambian de categoría atendiendo a nuevos significados que emergen. Mencionamos solo tres ejemplos que representan un porcentaje importante. 6. Género. Todos los motivos que mencionan de modo explícito el género de los niños se clasificaban en categoría identidad social, como "juega con las chicas", "es un chico", "pega a las chicas" o "es pesadito dando besos a algunas chicas". Sin embargo, se decidió sacar de esta categoría todas las conductas que van más allá del prejuicio asociado al género, como son las asociadas con el cuerpo de las chicas (besar, abrazar, chupar,...), indicativas de abuso sexual, así como expresiones 


\section{¿POR QUÉ NO NOS GUSTAN ALGUNOS COMPAÑEROS? RAZONES DE LOS ESCOLARES DE PRIMERO Y SEGUNDO CURSO DE EDUCACIÓN PRIMARIA}

como "pega a las chicas" o "es un chico y me puede hacer daño" que revelan violencia física. Ambas pasan a forman un bloque denominado violencia de género, dentro de la subcategoría intimidación. Se toma esta decisión para dar visibilidad a las conductas de violencia de género, y que no quedaran diluidas entre otros prejuicios o estereotipos o discriminación de género. 7. Malo. Las respuestas "es malo" o variaciones se clasificaban en intimidación. Se consideraba que era un expresión muy fuerte, y la asociábamos con es malo con la gente o mal-trata. Por paralelismo también se clasificaban en intimidación los motivos "dice cosas malas" o "hace cosas malas" y estas expresiones ya no nos parecían tan intensas o graves, y no estábamos seguros. Ello junto con que estas expresiones son muy frecuentes en niños de esta edad, nos hizo pensar que los niños no le atribuían un significado intimidatorio a la expresión "dice cosas malas" ni a "es malo", sino que podría ser una expresión general que denota mal comportamiento o mala conducta. Y entonces pasaron a clasificarse en la categoría conductas problemáticas, excepto cuando ser malo va claramente ligado a personas, como "me dice cosas malas" y "se porta mal con Adrián" o "hace cosas malas a la gente", que se clasifican en agresión verbal e intimidación, respectivamente. 8. Subcategorías en la categoría Conductas sociales y escolares problemáticas. En la categorización propuesta por García Bacete y cols. (2017) ya se realiza una cierta diferenciación, pero es ahora cuando emergen dos subcategorías para diferenciar cuando el incumplimiento de las normas es por violación (anti-norma) o por incompetencia (a-norma).

Con cada uno de estos aspectos se ha elaborado una guía de clasificación. La guía orienta la clasificación de los motivos en las categorías sustantivas, estableciendo criterios para diferenciar entre razones aparentemente iguales, o para decidir a qué categoría pertenece un determinado motivo en ausencia de mejores diferencias. Por ejemplo, los motivos que llevan la palabra pelea pueden tener significados diferentes y entonces se clasifican en categorías distintas: "Pelear" o "pelearse con" se clasifican en agresión física; "peleamos" o "nos peleamos" en identidad personal-desagrados; "jugar a peleas" en identidad personal-preferencias; y "meterse en peleas" en conductas problemáticas.

\section{Descripción de los motivos de desagrado}

Para cada categoría se aporta su definición, sus posibles fundamentos y ejemplos, y su porcentaje de frecuencias.

Categoría Conductas Agresivas ( $n=780 ; 30.1 \%$ ). Los investigadores están de acuerdo en que la razón principal del rechazo entre iguales son los comportamiento agresivos (Monjas et al., 2008). Se trata de conductas directas del niño rechazado que tienen la intención de causar dolor 0 daño personal o físico a otras personas, o que potencian la inseguridad y el miedo a poder sufrirlo. Leary (2001) se refiere a este comportamiento cuando afirma que rechazamos a las personas que percibimos como peligrosas. Desde la teoría de coordinación de las perspectivas sociales de Selman (2003), en los niños de 6 a 8 años las estrategias de resolución de conflictos centradas en uno mismo son las más habituales. Durante estas edades el niño descubre que hay otras maneras de ver el mundo, además de la propia, pero él sólo puede expresar y tratar de satisfacer sus propios deseos, lo que puede explicar por qué la agresión directa es tan frecuente.

En esta categoría distinguimos tres subcategorías. Las conductas de agresión gestual y verbal dirigidas a humillar, deteriorar la imagen o la reputación de otros. Estas conductas se agrupan en dos grandes polos: la agresión no verbal, expresada a través de gestos ("me pone mala cara") y el tono de voz ("nos gruñe), y las conductas que encajarían bajo la etiqueta de habla mal a alguien y se mete con alguien, como por ejemplo "me dice cosas feas", "insulta a todos", "se burla", "nos culpa", o formas de agresión relacional (por ejemplo "dice mentiras de mí", "me dice que los otros no son amigos míos"). En la segunda subcategoría se incluyen las conductas de agresión física. Estas conductas tienen el objetivo indisimulado de causar daño físico. La agresión se expresa de 
forma genérica o nombrando formas específicas de agresión ("siempre me hace daño", "pega a todos", "escupe", "me da patadas", me tira al suelo",...). Por último, encontramos las conductas de intimidación que tienen la intención de atemorizar a otros, provocar pérdida de control sobre lo que puede suceder, mediante el uso de amenazas, enfados y expresiones de ira, conductas de maltrato, abuso, y violencia de género ("amenaza", "se enfada mucho y rápido", "se porta mal conmigo", "hace llorar a los niños", "siempre me quiere besar y yo no quiero").

Categoría Dominancia $(n=91 ; 3.5 \%)$. La dominancia social es la competencia en un grupo 0 diada por el control de los recursos materiales y sociales (Hawley, 1999). Se dice que un niño o una niña es dominante cuando consigue que otros hagan lo que él/ella quiere. La dominancia social se asocia con jerarquía y estatus, características necesaria para el liderazgo y la organización, pero también con poder e influencia, imposición, control y superioridad, y entonces ya no gusta a los iguales y la penalizan porque perciben estas conductas como una influencia coercitiva, que reduce 0 impide la satisfacción de las necesidades de autonomía personal y reciprocidad en las interacciones sociales y escolares (Ochaita y Espinosa, 2012). Hatfield y Rapson (2011), desde la teoría de la igualdad, sostienen que el desequilibrio en las relaciones provoca emociones negativas, baja satisfacción y socava las relaciones. Leary (2001) afirma que es muy probable que las personas que se aprovechan de otros para sus propios fines sean rechazadas. Además, como señalan Abrams and Rudland (2008), en el contexto de los grupos hay que considerar el balance de oportunidades y amenazas desde la perspectiva de más de un actor. También se penaliza la sobrestimación de competencia y poder. De hecho la ilusión positiva que exhiben algunos niños agresivos y populares incrementa la agresión proactiva (Orobio, Brendgen, van Boxtel, Vitaro, \& Schaepers, 2007). Sin embargo, en estas edades la dominancia social todavía no es una conducta muy desagradable para los iguales (Monjas et al., 2008), posiblemente porque las jerarquías no están suficientemente establecidas, y la reciprocidad y la coordinación no son conductas normativas (Selman, 2003).

Las razones incluidas en esta categoría son aquellas que expresan desagrado hacia el ejercicio de poder coercitivo o impositivo ("es muy mandón", "ordena", "me quita las ideas"), la búsqueda de influencia sobre las personas, las relaciones y los recursos, con la intención que otros jueguen, hagan, vayan, hablen de esto 0 de aquello 0 con quien, según lo que él/ella desea ("me dice que no juegue con Laura", "no deja a Luis jugar con nosotros", "se juega a lo que ella quiere", "sólo quiere que juegue con ella"), o la sobrestimación de la propia competencia y estatus para ponerse en situación de superioridad y de acceso a privilegios de modo que se relega a los otros a situaciones inferiores y pérdida de autonomía ("se hace el chulo", "se cree el mejor").

Categoría Conductas Sociales y Escolares Problemáticas ( $n=575 ; 22.2 \%)$. Esta categoría pone el acento simultáneamente en los comportamientos sociales y escolares anti-normativos y a-normales del niño o la niña rechazados. Norma y grupo son los dos conceptos claves. El incumplimiento o desviación de la norma, en su doble acepción de normativa o normalidad, referida a reglas 0 a estándares y expectativas sociales y escolares, es el elemento diferenciador de las razones incluidas en esta categoría. Consecuentemente, el grupo, entendido indistintamente como conjunto de personas, categoría de desarrollo o parte de la sociedad, es el referente o garante del cumplimiento. Horn (2006) señaló que los niños y niñas y adolescentes se refieren constantemente a la contribución de los miembros del grupo a la continuidad y al funcionamiento del grupo como una razón importante para justificar el rechazo y la exclusión. Levine, Moreland y Hausmann (2005), desde la teoría del intercambio social, afirman que las personas y los grupos pueden interpretar que determinadas conductas son un coste para la relación, como cuando un miembro no aporta nada o poco, o perjudica al grupo (por ejemplo, quienes no siguen las reglas), o cuando sus interacciones y relaciones son atípicas (por ejemplo, conductas tímidas típicas). 


\section{¿POR QUÉ NO NOS GUSTAN ALGUNOS COMPAÑEROS? RAZONES DE LOS ESCOLARES DE PRIMERO Y SEGUNDO CURSO DE EDUCACIÓN PRIMARIA}

En la primera subcategoría encontramos conductas anti-normativas o comportamientos que van contra las normas escolares y sociales, de lo que está reglado y/o se considera apropiado (Levine et al., 2005). Estas conductas caen bajo la etiqueta de mala conducta, porque violan reglas reguladas por leyes y reglamentos o porque violan ideales o modelos de la sociedad 0 grupos de los que se es miembro. La expresión de desagrado hacia estas conductas destaca lo prohibido y refuerza las normas legales y morales. Se considera que quienes realizan estas conductas son miembros desleales con el grupo y han de ser sancionados por sus conductas antisociales (Abrams, \& Rutland, 2008). Se incluyen conductas contra la propiedad ("roba", "rompe", "raya",...), las reglas ("hace trampas", ..., "gamberro", "se porta mal", "es malo",...), la autoridad ("no hace caso", "se enfrenta a la maestra", "le riñen",...), los buenos modales o la buena educación ("es guarro", "mocoso", "hace cosas malas que no me gustan", "dice palabrotas",...), y la confianza ("se chiva", "miente", "engaña", "se copia", "se mete en todo",...).

En una segunda categoría encontramos conductas a-normales o conductas que se desvían, por defecto 0 por exceso, de las conductas, logros o estándares de desarrollo o escolares que se consideran norma-les en una determinada edad, etapa educativa o grupo. Se refieren a déficits o excesos en las habilidades sociales y escolares necesarias para el éxito relacional y educativo. Ahora no se trata de ataques a la norma, sino de conductas que no se ajustan a la norma-lidad. Se considera que los alumnos que realizan estas conductas son miembros incompetentes que no contribuyen al funcionamiento del grupo y al éxito escolar y social (Leary, 2001), que son una carga para el grupo (Wesselmann, Wirth, Pryor, Reeder, \& Williams, 2013). Se incluyen conductas que delatan que el niño o la niña es incompetente social o escolarmente, porque sus habilidades sociales no responden a lo que se espera ("es antipático", "no me deja el sacapuntas", "es mal compañero"), no controla la propia conducta, por exceso ("es un poco bruto", "se enfada", "está loca", "se ríe por nada"), o por defecto ("habla muy bajito", "es un llorica", "tarda mucho"), o porque es incompetente como jugador o como alumno ("hace cosas de niños más pequeños", "no juega bien a los juegos que hacemos", "no estudia", "hace la letra muy mal").

Categoría Molestias al Bienestar $(n=441 ; 17 \%)$. Se trata de conductas inoportunas y repetitivas del rechazado, generalmente de baja intensidad, que interfieren en las condiciones de bienestar 0 de realización de actividades del rechazador o su grupo, lo que acaba provocando malestar, aburrimiento y sensación de ineficiencia e ineficacia. Monjas y cols. (2008) hablan de comportamientos que revelan inmadurez y falta de atención, que agobian o resultan molestos para los otros. La investigación educativa se refiere a estos comportamientos como disruptivos y los identifica como la principal fuente de la mayoría de los problemas de convivencia en las aulas (Levin, \& Nolan, 2014). Se incluyen conductas que producen molestias y fastidios, distracciones e interrupciones, inoportunidad e insistencia, cansancio, agobio y enfado, aburrimiento e inutilidad ("molesta", "siempre está interrumpiendo", "es un pesado", "nos agobia todo el rato", "me hace enfadar", "es aburrido", "no hace cosas divertidas", "dice tonterías").

Categoría Identidad Personal ( $n=375 ; 14.5 \%$ ). En esta categoría, el sujeto expresa que sus gustos son distintos de los de aquellos a quienes rechaza, bien porque cada uno tiene sus propias preferencias, bien expresando desagrados, exclusiones o conflictos, de modo unidireccional o recíproco. Leary (2001) señala que las elecciones relacionales revelan preferencias personales, basadas en agrados 0 desagrados personales. Nucci (2001) sugiere que hay cuestiones, como la elección de amigos o de hobbies, que los sujetos consideran que son prerrogativas de su exclusiva competencia, reflejo de su propia identidad y autonomía. En esta categoría se destaca el componente relacional, poniendo el foco no en las conductas del rechazado sino en la relación entre ambos.

En esta categoría encontramos un conjunto de razones que indican que las personas tienden a 
relacionarse con quienes tienen gustos o realizan actividades similares (Gifford-Smith, \& Brownell, 2003), y como consecuencia otros quedan al margen (Leary, (2001). Desde la perspectiva de los grupos, existe evidencia de que los niños cuando se les exige tomar decisiones, expresar las preferencias 0 asignar recompensas entre varios grupos muestran un fuerte sesgo hacia su propio grupo (Nesdale, 2011). En esta subcategoría el niño expresa sus preferencias, gustos, aficiones, o elecciones de personas, juegos, relaciones o amistades o las de su grupo ("nos gusta jugar al futbol", "me gusta estar con Roberto", "juego con mis amigos", "jugamos a pilla-pilla") o las de aquel 0 aquellos que le desagradan ("juega a otras cosas", "juega con sus amigos", "es amiga de Cristina", "siempre está con David").

La segunda subcategoría está formada por motivos en los que rechazador o rechazado (o lo que cree el rechazador que piensa, dice o hace el rechazado) declaran la voluntad, unidireccional o recíproca, de no tener vínculo amistoso, relacional o lúdico entre ellos, sus amigos o sus grupos. El desagrado desde el rechazador al rechazado es una expresión de su autonomía personal en forma de preferencias o elecciones negativas. Los motivos pueden expresar diferente intensidad, desde el desagrado y la aversión, a la exclusión y la mala relación y conflicto ("me cae mal", "no me gustan sus amigos", "no me gusta a lo que juega", "va con niñas que yo no conozco", "no me apetece estar con él", "no le busco", "no me llevo bien con él"). En sentido inverso, cuando el rechazador percibe desagrado o conductas de exclusión hacia él o su grupo, entonces como revancha reacciona con desagrado 0 exclusión hacia el rechazado (Leary, 2001) ("me tiene envidia", "no me deja jugar con él", "no me invitó a su cumpleaños", no quiere juntarse con nosotros", "cuando me ve se va"). Por último, encontramos expresiones de desagrados o conflictos mutuos ("no nos gustan las mismas cosas", "nos peleamos"). En esta subcategoría también se incluyen las reputaciones negativas que el rechazador atribuye al rechazado por conductas pasadas o que solo han ocurrido una vez ("decía palabrotas"; "un día me rompió una pulsera"), que revela la fuerza de las reputaciones que actúan como si de conductas reales se tratasen con el agravante de que no se pueden contrastar o negar (Mikami et al., 2010).

Categoría Identidad Social $(n=90 ; 3.5 \%)$. En los motivos de esta categoría se expresa un desagrado hacia otros niños o niñas sobre la base de su pertenencia a un grupo o categoría social a la que se le atribuyen rasgos o conductas negativas ("es una chica") o por la realización de actividades atribuidas a estos mismos grupos ("juega a cosas de chicas"), en ausencia de otras razones más específicas. Estas motivaciones pueden explicarse a partir de los conceptos de estereotipo, prejuicio y discriminación (Mulvey, Hitti, \& Killen, 2010). Desde la teoría de desarrollo del intergrupo, Nesdale (2011) señala que bajo determinadas condiciones el favoritismo hacia el propio grupo se convierte en estereotipo o prejuicio (p.e., cuando el niño establece una fuerte identificación con el grupo, o los miembros se sienten amenazados por miembros del otro grupo. Sobre esta base, Leary (2001) informa que ciertos grupos son potencialmente más rechazados que otros.

Los prejuicios de grupo que han emergido en nuestro estudio son los que se derivan de la pertenencia a otro sexo ("nunca juega con las chicas"), territorio o etnia ("es de otro pueblo", "no me gusta la gente negra"), clase social ("es pija"), ser de otro grupo ("es del Betis"), o de edades diferentes ("va con niñas más mayores"), o ser nuevo ("no estaba en infantil"), o tener alguna discapacidad o rareza ("camina muy raro"), o aspecto físico desagradable ("es feo").

Categoría Falta de Contacto o de Familiaridad ( $n=179 ; 6.9 \%$ ). Existe otro conjunto de motivos que representan la ausencia o escasez de intercambios y de conocimiento, de actividades y elecciones entre rechazador y rechazado, por lo que no pueden estar ni en la supracategoría de conductas ni en la de preferencias. Allport ya afirmó en 1954 que, en ausencia de conductas negativas, la falta de contacto 0 de familiaridad puede llevar al rechazo. En esta categoría "bisagra" se incluyen moti- 


\section{¿POR QUÉ NO NOS GUSTAN ALGUNOS COMPAÑEROS? RAZONES DE LOS ESCOLARES DE PRIMERO Y SEGUNDO CURSO DE EDUCACIÓN PRIMARIA}

vos que reflejan la indiferencia hacia los otros o la baja aceptación, bien por la inercia social para seguir haciendo lo que ya se hace 0 se prefiere o por el desinterés hacia las actividades y habilidades de otros (Bourdieu, 1985), bien por el miedo y la desconfianza hacia lo que es desconocido 0 no familiar (Gifford-Smith, \& Brownell, 2003). Leary (2001) se refirió a ello diciendo que el rechazo puede ser el resultado de la imposibilidad de aceptar a todos o del fracaso. Revela un fracaso por ambas partes en apreciar la relación con otra persona (Leary, 2001) y por inmovilismo y pasividad, lo que era neutro acaba siendo negativo sin mediar razones. Así encontramos motivos que expresan desconocimiento ("no lo conozco"), poco o ningún contacto ("no viene conmigo"; "me hablo poco con él"), que no comparten juegos ("no juega conmigo"; "nunca juego con él), ni amistad ("no es amigo"; "no somos buenos amigos").

Categoría No sabe- No contesta $(n=62 ; 2.4 \%)$. Hay niños que no dan ninguna razón para sus nominaciones negativa o dan respuestas del tipo "no lo sé", "no sé por qué", "no me acuerdo", y "porque no". Puede ser que no deseen revelar sus motivos, o que no sepan expresarlos, o que no se sientan a gusto nominando, o que sigan la inercia de otros compañeros de la clase,... Independientemente de cual pueda ser la explicación, se puede concluir que para que no te guste un compañero no hacen falta razones, o hacerlas públicas.

\section{DISCUSIÓN}

En este estudio hemos avanzado en la comprensión de las razones de desagrado a través de las explicaciones que los rechazadores dan sobre por qué no les gustan algunos compañeros/as. Los desagrados son algo común en las relaciones, son funcionales, pero también interrumpen los contactos (Redorta et al., 2006). Solo conociéndolos se puede actuar. ¿Qué nos enseña la taxonomía de razones del desagrado?

1. Que existe una gran variabilidad de motivos de desagrado. En el estudio han emergido 12 subcategorías excluyentes de motivos de desagrado explicadas por tres motivaciones que subyacen a las conductas y atracciones. Conduce al rechazo lo que se interpreta como amenaza 0 ataque a la seguridad emocional y física, a la autonomía, a las reglas y lo que es normal, al bienestar, a la identidad personal y social, o lo desconocido. Conocer las razones de desagrado incrementa las posibilidades de comprender tanto a los niños/as rechazados como a los/las rechazadores, comprender sus intercambios sociales o su ausencia. Conocer los motivos concretos por los que a un alumno no le gustan algunos compañeros, o por los que un alumno no gusta a sus compañeros ofrece pistas valiosísimas para saber por dónde empezar a intervenir (Di Napoli, 2017; García Bacete, Marande, \& Mikami, 2019a). Por ejemplo imaginemos que de un alumno se dice que pega y que hace daño a los demás, entonces puede ser aconsejable enseñarle a practicar métodos alternativos de solución de conflictos, o que varios alumnos dicen que no les gusta una compañera porque es chica y fea, en este caso se realizarían en el aula ejercicios para combatir los procesos de categorización social y atribución que operan sobre el género y la atracción física. Pero, además, conocer que la mayor parte de estos motivos están presentes en casi todas las aulas invita al profesor a anticipar y planificar la organización y el curriculum del aula teniendo en cuenta los motivos de los alumnos para relacionarse, establecer antagonismos o no interactuar-(Bukowski, Laursen, \& Rubin, 2018). Por ejemplo, saber que la indiferencia es un motivo omnipresente que además se incrementa con la edad es un argumento pedagógico para que los profesores creen ambientes seguros y de respeto en los que se promueven relaciones igualitarias y de cooperación entre compañeros diferentes (Allport, 1954), que ayuden a los alumnos a vencer su inercia y sus conductas de autoprotección (Hatfield, \& Rapson, 2011). Máxime cuando se sabe que el grupo de pares presenta sesgos de reputación que les predispone a mantener sus desagrados (Mikami et al., 2010). 
2. Que los desagrados son evaluaciones elaboradas por los emisores. Las motivaciones de identidad e indiferencia son claramente externas a los alumnos rechazados. Las preferencias e identidades, la falta de contacto o el desinterés son construcciones de los emisores. Pero, también lo son las razones conductuales, puesto que son los emisores quienes interpretan qué conductas y de quienes conllevan un coste personal o grupal. De esta forma se pone el foco en las bases cognitivas de las relaciones sociales (Selman, 2003). Así pues, conocer la perspectiva de los rechazadores es indispensable, y en consecuencia se debe intervenir fundamentalmente sobre los emisores y sus evaluaciones para que aprendan a ser conscientes de sus prejuicios, a ser más flexibles en sus juicios, y a descubrir atributos y conductas positivas en los niños que no les gusta (García Bacete et al., 2019a).

3. Que muchas de las nominaciones negativas y razones son encubiertas. Los motivos no sabe/no contesta, los expresados en pasado, o los que señalan falta de familiaridad van en esta dirección. Los numerosos errores en la identificación de a quienes no gustamos lo confirma (García Bacete et al., 2019b). Este carácter encubierto refuerza el carácter interpretativo de los desagrados, pero además recomienda que los desagrados se hagan visibles de forma clara y descriptiva. Dada la tendencia a ocultar los desagrados en los encuentros cara a cara como mecanismo de autoprotección (Bellmore, \& Cillessen 2003) es necesario que alumnos y profesores se den cuenta de sus motivos de desagrado hacia otros alumnos y que se les enseñe formas adecuadas de expresarlos. Equilibrar la balanza entre lo que se dice y lo que se oculta y enseñar cómo dar feedback negativo es de aplicación en este caso. Mikami y Mercer (2017) animan a dar feedback positivo de forma pública y a expresar los desagrados negativos de forma discreta y acompañados con expresiones positivas que orienten sobre conductas y actitudes alternativas. Si bien hay que ser cautos. Por ejemplo, García Bacete et al. (2019b) afirman que para los alumnos rechazados subestimar los desagrados tiene un carácter protector.

4. Que los desagrados tienen naturaleza grupal. Las razones que tienen como referente a un grupo son una mayoría y se distribuyen por todas las categorías. En el caso de la categoría conductas problemáticas que informa de dinámicas intragrupales y de la categoría identidad social sobre las dinámicas entregrupos el carácter grupal es algo evidente (Nesdale, 2011; Juvonen, 2018). Los grupos se estructuran sobre la base de los afectos y conductas entre sus miembros (Bukowski et al., 2018; Juvonen, 2018). Además, el uso frecuente en las razones del nombre de compañeros, 0 de expresiones como amigos o nosotros, y de situaciones y normas conocidas revela el carácter altamente significativo y grupal del rechazo y su resistencia al cambio. Todo ello junto con que el $95 \%$ de los alumnos son emisores de desagrados y que ser miembro de un mismo grupo-aula es muy estable en el contexto español habla de la necesidad de intervenciones universales dirigidas a todos los alumnos, y a hacerlo de forma significa, continuada, global,... porque tiene que servir para las relaciones presentes y futuras (García Bacete y cols., 2019a).

5. Permiten programar intervenciones preventivas. La mayoría de investigadores defienden las actuaciones preventivas, pero muy pocos las practican. Indagar en las razones permite identificar el germen de conductas negativas que pueden progresar en gravedad y frecuencia si no se interviene. Prestar la atención necesaria a los desagrados en estas edades puede ayudar a comprender y combatir situaciones de acoso y victimización puesto que los desagrados pueden ser la semilla que da origen a las conductas de acoso, ya que están presentes en el $80 \%$ de los casos de bullying (Schuster, 2001). La taxonomía permite darse cuenta que los niños de 6-7 años expresan razones que denuncian nítidamente conductas de violencia de género ("quiere que enseñe las bragas", "pega a las chicas"). Desarrollar actividades dirigidas a combatir los estereotipos de género y realizar agrupamientos mixtos en las aulas puede ayudar a hacer frente a esta lacra social (Rose, \& Smith, 2018). 


\section{¿POR QUÉ NO NOS GUSTAN ALGUNOS COMPAÑEROS? RAZONES DE LOS ESCOLARES DE PRIMERO Y SEGUNDO CURSO DE EDUCACIÓN PRIMARIA}

Otro ejemplo, la dominancia es poco frecuente en estas edades, pero sabemos que aumenta con la edad y que cursa en muchos casos con agresividad (Monjas et al., 2008). Como se ha visto la dominancia se asocia con poder coercitivo, influencia y sobrestimación de las competencias, que conlleva que otros niños sientan pérdida de autonomía. Se trata pues de que los niños tengan oportunidades para hacer elecciones, contribuir al bien del grupo y ajustar sus percepciones. Ello se puede conseguir promoviendo actividades curriculares en las que los niños puedan ejercer roles de liderazgo y de organización de actividades, que los niños hablen sobre sus deseos y que defiendan su derecho a participar y a ser diferentes, normalicen la reciprocidad y los intercambios igualitarios, que aprendan a ser observadores asertivos que denuncien egoísmos y conductas desleales,..., en suma que mejoren sus competencias socioemocionales.

6. Que de forma global los desagrados y los rechazos se pueden interpretar como una actitud contra la diferencia, contra los diferentes, los imperfectos, los que comenten errores, los que rompen normas, los que tienen gustos diferentes, los que quieren organizar nuestra vida o carecen de competencias, los desconocidos,... Las categorías identidad social e indiferencia son una prueba incuestionable. Los grupos de pares tienden a elegir a quienes comparten atributos, actitudes 0 conductas similares (Gifford-Smith, \& Brownell, 2003; Nesdale, 2011) y tienden a desconfiar y devaluar socialmente a los compañeros de clase 0 de otras clases que perciben como diferentes (Chang, 2004). Los desagrados son estados de ánimos que predisponen en contra de la inclusión. Y es tan así que las diferencias se penalizan, incluso cuando son inofensivas, como cuando el desagrado viene motivado por ser de otro grupo 0 tener gustos diferentes (García Bacete et al., 2017). La escuela no puede prohibir los desagrados o hacer que no se expresen. El papel prioritario de la escuela consiste en crear escenarios que incrementen los contactos y la familiaridad entre el alumnado y entre las familias y promuevan comportamientos y actitudes inclusivas entre los miembros de la comunidad (Juvonen, 2018).

El papel del profesorado es relevante en la enseñanza y el modelado de habilidades socioemocionales para expresar, comprender y regular las emociones, animar las conductas inclusivas en el aula y combatir sesgos de reputación y prejuicios, tomar conciencia de sus preferencias y expectativas y reflexionar sobre la forma de mejorar sus relaciones con los alumnos, y apoyar a las familias en las relaciones con sus hijos y en la enseñanza de habilidades para hacer amigos, en la solución cooperativa de problemas y en la provisión de escenarios de ocio y aprendizaje seguros (García Bacete et al., 2019a). En términos generales el profesorado tiene que desarrollar intervenciones educativas de carácter universal y curricular, con todo el alumnado en el aula, especialmente centradas en la condición de emisores de desagrados, junto con intervenciones focalizadas en diadas y alumnos concretos que lo necesiten (emisores y receptores).

Con este estudio la taxonomía elaborada por García Bacete et al. (2017) ha mostrado su solidez, no obstante deseamos seguir verificando el principio de modificabilidad usando muestras de niños mayores y de otras culturas. También estamos interesados en analizar los motivos desde una perspectiva de género que informe sobre lo que no les gusta a las chicas y sobre lo que no gusta de las chicas. Una última línea de futuro conlleva estudiar el carácter relacional de los motivos a través del análisis de desagrados que se dan entre diadas, así como verificar si formar parte de una misma aula influye en los motivos de desagrado que se emiten en ese grupo.

\section{AGRADECIMIENTOS:}

Este trabajo ha sido posible a las ayudas concedidas al primer autor por el Ministerio de Economía y Competitividad del Gobierno de España (EDU2012-35930) y por la Universidad Jaume I (P1-1A2012-04). 


\section{REFERENCIAS BIBLIOGRÁFICAS}

Abrams, D., \& Rutland, A. (2008). The development of subjective group dynamics. In S. R. Levy \& M. Killen (Eds.), Intergroup relations and attitudes in childhood through adulthood (pp. 47-65). Oxford, UK: Oxford University Press.

Allport, G. W. (1954). The Nature of Prejudice. Reading, MA: Addison-Wesley.

Bellmore, A. D., \& Cillesen, A. H. N. (2003). Children's meta-perceptions and meta-accuracy of acceptance and rejection by same-sex and other-sex peers. Personal Relationships, 10, 217233. doi: $10.1111 / 1475-6811.00047$.

Berger, C., \& Dijkstra, J. K. (2013). Competition, envy, or snobbism? How popularity and friendships shape antipathy networks of adolescents. Journal of Research on Adolescence, 23, 586-595. doi: 10.1111/jora.12048 1

Bierman, K. L. (2004). Peer rejection: developmental processes and intervention strategies. Nueva York, NY: The Guilford Press.

Bisquerra, R. (2015). Universo de emociones. Valencia: PalauGea.

Bourdieu, P. (1985). The social space and the genesis of groups. Social Science Information. 24, 195-220. doi: 10.1177/053901885024002001

Bukowski, W. M., Laursen, B., \& Rubin, K. H. (2018, $2^{\circ}$ Ed.). Handbook of peer interactions, relationships, and groups. New York, NY: Guilford Press.

Bukowski, W. M., Sippola, L., Hoza, B., \& Newcomb, A. F. (2000). Pages from a sociometric notebook: an analysis of nomination and rating scale measures of acceptance, rejection, and social preference. In A. H. N. Cillessen \& W. M. Bukowski (Eds.), Recent Advances in the Measurement of Acceptance and Rejection in the Peer System (pp, 11-26). San Francisco, CA: Jossey-Bass.

Bukowski, W. M., Motzoi, C., \& Meyer, F. (2009). Friendship as process, function, and outcome. In K. H. Rubin, W. M. Bukowski, \& B. Laursen (Eds.), Social, emotional, and personality development in context. Handbook of peer interactions, relationships, and groups (pp. 217-231). New York, NY, US: The Guilford Press.

Carrero, V. E., Soriano, R., y Trinidad (2012). Teoría fundamentada "Grounded Theory": El desarrollo de la teoría desde la generalización conceptual. Madrid: Centro de Investigación Sociológica. Colección Cuadernos metodológicos nำ37.

Casas, F. (2010). Indicadores sociales subjetivos \& bienestar en la infancia \& la adolescencia. In G. González-Bueno, M. von Bredow, \& C. Brecedóniz (Eds.), Propuesta de un sistema de indicadores sobre el bienestar infantil en España (pp. 34-51). Madrid: UNICEF-España.

Chang, L. (2004). The role of classroom norms in contextualizing the relations of children's social behaviors to peer acceptance. Developmental Psychology. 40, 691-702. doi: 10.1037/00121649.40.5.691

Doosje, B., Spears, R., \& Ellemers, N. (2002). Social identity as both cause and effect: the development of group identification in response to anticipated and actual changes in the intergroup status hierarchy. British Journal of Social Psychology, 41, 57-76. doi: 10.1348/014466602165054

Elig, T. W., \& Frieze, I. H. (1979). Measuring causal attributions for success and failure. Journal of Personal and Social Psychology 37, 621-634. doi: 10.1037/0022-3514.37.4.621

Feinberg, M. R., Smith, M., \& Schmidt, R. (1958). An analysis of expressions used by adolescents of varying economic levels to describe accepted and rejected peers. Journal of. Genetic Psychology, 93, 133-148. doi: 10.1080/00221325.1958.10532411

García Bacete, F. J., Carrero, V. E., Marande, G., \& Musitu, G. (2017). Understanding rejection between first and second grade elementary students through reasons expressed by rejecters. Frontiers in Psychology, 8, 1-13. doi: 10.3389/fpsyg.2017.00462 


\section{¿POR QUÉ NO NOS GUSTAN ALGUNOS COMPAÑEROS? RAZONES DE LOS ESCOLARES DE PRIMERO Y SEGUNDO CURSO DE EDUCACIÓN PRIMARIA}

García Bacete, F. J., Marande, G., \& Mikami, A. Y. (2019a, in press). Evaluation of a Multi-component and Multi-agent Intervention to Improve Classroom Social Relationships among Early Elementary School-Age Children. Journal of School Psychology,

García Bacete, F. J., Marande, G., Schneider, B, \& Cillessen, A. H. N. (2019b). Children's Awareness of Peer Rejection and Teacher Reports of Aggression. Psychosocial Intervention, 28 (1), 37-47. doi: $10.5093 /$ pi2018a25

Gifford-Smith, M. E., \& Brownell, C. A. (2003). Childhood peer relationships: Social acceptance, friendships, and peer networks. Journal of School Psychology, 41, 235-284. doi:10.1016/S0022-4405(03)00048-7.

Hatfield, E., \& Rapson, R. L. (2011). Equity theory in close relationships. In P. A. M. Van Lange, A. W. Kruglanski \& E. T. Higgins (Eds.), Handbook of Theories of Social Psychology (pp. 200-217). London: Glyph International.

Hayes, D. S., Gershman, E. S., \& Halteman, W. (1996). Enmity in males at four developmental levels: Cognitive bases for disliking peers. The Journal of Genetic Psychology: Research and Theory on Human Development, 157, 153-160. doi:10.1080/00221325.1996.9914853

Horn, S. (2006). Group status, group bias, and adolescents' reasoning about treatment of others in school contexts. International Journal of Behavioral Development, 30, 208-218. doi: $10.1177 / 0165025406066721$

Juvonen, J. (2018). The Potential of Schools to Facilitate and Constrain Peer Relationships . In K. H. Rubin, W. M. Bukowski, \& B. Laursen (Eds.), Social, emotional, and personality development in context. Handbook of peer interactions, relationships, and groups (pp. 217-231). New York, NY, US: The Guilford Press.

Killen, M., Mulvey, K. L., \& Hitti, A. (2013). Social exclusion: A developmental intergroup perspective. Child Development, 84(3), 772-790. doi:10.1111/cdev.12012.

Leary, M. R. (2001). Toward a Conceptualization of Interpersonal Rejection.

Leary, M. R. (2005). Varieties of interpersonal rejection. In K. D. Williams, J. P. Forgas \& W. von Hippel (Eds.), The social outcast: Ostracism, social exclusion, rejection, and bullying, (pp. 3551). New York: Psychology Press.

Levin, J., \& Nolan, J. (2014). Principles of Classroom Management: A Professional Decision-Making Model. Boston: Pearson.

Levine, J. M., Moreland, R. L., \& Hausmann, L. M. R. (2005). Managing group composition: Inclusive and exclusive role transitions. In D. Abrams, J. M. Marques \& M. A. Hogg (Eds.), The social psychology of inclusion and exclusion (pp. 137-160). Philadelphia: Psychology Press.

McDougall, P., Hymel, S., Vaillancourt, T., and Mercer, L. (2001). The consequences of childhood peer rejection. In M. R. Leary (Ed.), Interpersonal Rejection (pp., 213-247). New York, NY: Oxford University Press.

Mikami, A.Y., Lerner, M. D., \& Lun, J. (2010). Social context influences on children's rejection by their peers. Child Development Perspectives, 4(2), 123-130. doi: 10.1111/j.17508606.2010.00130.x

Mikami, A. Y., \& Mercer, S. H. (2017). Teacher behaviors toward children with AttentionDeficit/Hyperactivity Disorder predict peers' initial liking and disliking impressions in a summer camp setting. Journal of Social and Clinical Psychology, 36, 506-534.

Moliner, B. (2018). Do rejected students know that they are? According to the rejector's point of view. Trabajo Fin de Grado de Psicología. Universitat Jaume I. Castellón.

Monjas Ma. I., Sureda, I., y García Bacete, F. J. (2008). ¿Por qué los niños y niñas se aceptan y se rechazan? Cultura \& Educación, 20(4), 479-492. doi: 10.1174/113564008786542181 
Moreno, J. L. (1934). Who shall survive? A new approach to the problem of human interrelations. Washington, D.C.: Nervous and Mental Disease Publishing Co

Mulvey, K. L., Hitti, A., \& Killen, M. (2010). The development of stereotyping and exclusion. Wiley Interdisciplinary Reviews: Cognitive Science, 1, 597-606. doi: 10.1002/wcs.66

Nesdale, D. (2011). Social groups and children's intergroup prejudice: Just how influential are social group norms? Anales de Psicología, 27(3), 600-610.

Nucci, L. P. (2001). Education in the moral domain. Cambridge, England: Cambridge University Press. doi: 10.1017/CB09780511605987

Ochaita, E. y Espinosa, M. A. (2012). Los Derechos de la Infancia desde la perspectiva de las necesidades. Educatio Siglo XXI, 30 (2), 25-46.

Orobio de Castro, B., Brendgen, M., van Boxtel, H., Vitaro, F., \& Schaepers, L. (2007). "Accept me, or Else": Disputed overestimation of social competence predicts increases in proactive aggression. Journal of Abnormal Child Psychology, 35, 165-178.

Redorta, J., Obiols, M., \& Bisquerra, R. (2006). Emoción \& conflicto. Aprender a manejar las emociones. Barcelona: Paidós.

Rose, A. J., \& Smith, R. L. (2018). Gender and Peer Relationships. In W. M. Bukowski, B. Laursen, \& K. H. Rubin (Eds.), Handbook of peer interactions, relationships, and groups, (pp. 571-589). New York, NY: Guilford Press.

Schuster, B. (2001). Rejection and victimization by peers. In J. Juvonen \& S. Graham (Eds.), Peer harassment in school: The plight of the vulnerable and victimized (pp., 290-309). New York, NY: Guilford.

Selman, R. (2003). The Promotion of Social Awareness: Powerful Lesson from Partnership of Developmental Theory and Classroom Practice. New York, NY: Russell Sage Foundation.

Smith, G. D. (1950). Sociometric study of best-liked and least-liked children. Elementary School Journal, 51, 77-86. doi: 10.1086/459206

Valles, A., y Valles, C. (2000). Inteligencia emocional. Aplicaciones educativas. Madrid: EOS.

Wesselmann, E. D., Wirth, J. H., Pryor, J. B., Reeder, G. D., y Williams, K. D (2013) When Do We Ostracizem? Social Psychological and Personality Science 4(1) 108-115. 
